EESTI NSV TEADUSTE AKADEEMIA TOIMETISED. 29. KOIDE

FUOSIKA * MATEMAATIKA. 1980, NR. 4

ИЗВЕСТИЯ АКАДЕМИИ НАУК ЭСТОНСКОИ ССР. ТОМ 29

ФИЗИКА * МАТЕМАТИКА. 1980, № 4

А. КОППЕЛЬ, Т. ЛЕМБЕР

удк $530.12: 531.51$

\title{
О НЕРЕЛЯТИВИСТСКОМ АНАЛИЗЕ МЕТОДОВ ГЕНЕРИРОВАНИЯ АКСИАЛЬНО-СИММЕТРИЧНЫХ СТАЦИОНАРНЫХ РЕШЕНИЙ УРАВНЕНИЙ ЭЙНШТЕЙА
}

\author{
(Представил П. Кард)
}

\section{Введение}

Данная работа является непосредственным продолжением статьи [ $\left.{ }^{1}\right]$. Нами поставлена задача вывести основные формулы т. н. нерелятивистского (НР) анализа некоторых конкретных методов генерирования новых аксиально-симметричных (AC) стационарных решений уравнений Эйнштейна. Рассмотрим алгоритмы либо вида * $(1.9-1.10)$

$$
\mathcal{E}^{\prime}=F \varepsilon, \quad W^{\prime}=W, \quad H=1,
$$

либо вида

$$
\check{\lambda}^{\prime}=\check{\chi} \check{\chi}, \quad(\operatorname{det} \check{\chi})^{1 / 2}=H=1,
$$

где комплексный функционал $F$ или действительная $2 \times 2$-матрица $\check{\chi}$ определяются конкретным методом генерирования новых решений. Meтодом типа (1) является, например, метод Нейгебауэра $\left[{ }^{2,3}\right]$, а методом типа (2) - метод Белинского и Захарова [ $\left.{ }^{4}\right]$. Таким образом, наряду с парой $(\mathcal{E}, W)$ в качестве первичной характеристики поля принимается здесь матрица

$$
\check{\lambda}=-\lambda\left(\begin{array}{cc}
-\mathcal{E}_{+} \mathcal{E}_{-} & A \\
A & 1
\end{array}\right)=\left(\lambda_{B D}\right)
$$

где $B, D, \ldots=3,4 ; \mathcal{E}_{ \pm}=\lambda^{-1} W \mp A$, а $\lambda, A$ и $W-$ инвариантные характеристики поля, определяемые по формулам (1.2). Для 4-мерного описания данных релятивистских полей пользуемся локальными ортореперами Минковского, в которых метрическая форма имеет вид $\left(x^{4}=c x^{0}\right)$

$$
\begin{array}{cl}
d s^{2}=\lambda-1 \overline{d l^{2}}-c^{2}\left(\omega^{(0)}\right)^{2} \equiv\left(\omega^{(1)}\right)^{2}+\left(\omega^{(2)}\right)^{2}+\left(\omega^{(3)}\right)^{2}-c^{2}\left(\omega^{(0)}\right)^{2}, \\
\omega^{(1)}=\lambda^{-1 / 2} \mathrm{e}^{\mu+v} d x^{1}, \quad \omega^{(2)}=\lambda^{-1 / 2} \mathrm{e}^{\mu+\nu} d x^{2}, \\
\omega^{(3)}=\lambda^{-1 / 2} W d x^{3}, \quad \omega^{(0)}=\lambda^{1 / 2}\left[c^{-1} A d x^{3}+d x^{0}\right],
\end{array}
$$

а для 3-мерной трактовки имеем евклидовы 3 -пространства $V_{3}$, где метрическая форма имеет вид (1.8), т. е. $d l^{2}={\overline{d l^{2}}}_{\jmath \mu=0}$.

* Здесь и ниже ссылки на формулы [1] обозначаются двумя цифрами, причем первая цифра 1 есть номер той работы. 
В первом разделе приведем некоторые основные формулы НР анализа, во втором применим их в общем случае алгоритмов (1) и (2), а в третьем рассмотрим метод Нейгебауэра $\left[{ }^{2,3}\right]$, задавая характерный для этого метода функционал $F$ в определенном новом виде, формулы которого приведены в Приложении.

\section{Основные формулы}

Метод анализа релятивистских величин и соотношений на основе построения НР аналогов (НР анализ) заключает в себе разные уровни и аспекты $\left[{ }^{5-7}\right]$. При анализе методов генерации решений уравнений Эйнштейна естественно прежде всего рассматривать задачу изучения условий, при которых новому решению вообще соответствуют НР модели гравитационных полей, представляющие физический интерес. Это можно делать как на основе изучения 4-мерных НР геометрических моделей, так и на основе анализа 3-мерных аспектов получаемых НР пределов. Далее, на базе получаемых НР моделей можно приступить к выяснению физического смысла первых релятивистских поправок новых решений.

Если не ставить задачу изучения влияния возможных координатных преобразований на НР предел (анализ такого воздействия является особой задачей), 'т. е. если считать «координатный фон» $\left\{x^{v}\right\}$ фиксированным («изучаемое решение уже дано в определенной $\bar{G}^{r}$-системе» $[5,6])$, то в рассматриваемом АС стационарном случае для существования НР (как ньютоновых, так и неньютоновых) искривленных моделей пространства-времени должны иметь место следующие разложения для инвариантных полевых функций (1.2) в базисных 1-фор-
$\max (5):$

$$
\lambda=1+\stackrel{[2]}{\lambda} \eta^{2}+O\left(\eta^{3}\right), \quad A=\stackrel{[1]}{A} \eta+O\left(\eta^{2}\right), \quad W=\stackrel{[0]}{W}+O(\eta) .
$$

Здесь $\eta=c^{-1} v_{N}, v_{N}$ - определенный характеристический НР параметр размерности скорости. Формулы (6) определяют в свою очередь возможные разложения потенциала $\mathcal{E}$ и матрицы $\check{\lambda}(3)$. Далее потребуем, чтобы для новых решений в формулах (1)-(2) имели место разложения (6), дающие непосредственно в пределе $\eta \rightarrow 0$ отыскиваемые НР аналоги. Отсюда следует, что как $\mathcal{E}^{\prime}$, так и $\check{\lambda}^{\prime}$ должны быть аналитическими функциями параметра $\eta$ при $\eta \rightarrow 0$, причем всегда должны выполняться условия

$$
\stackrel{[0]}{\mathcal{E}^{\prime}}=1, \quad \operatorname{Re}{\stackrel{[1]}{\mathcal{E}^{\prime}}}^{\prime}=0
$$

и соответствующие дополнительные условия для $\grave{\lambda}^{\prime}$.

Для 3-мерной трактовки АС стационарных НР предельных гравитационных полей получается предельное евклидово $\stackrel{(0)}{V}$, где координаты определяются предельным видом метрической формы (1.8) 3-пространства $V_{3}$, т. е. пределом функции $W$. Вопрос о влиянии выбора конкретной гармонической функции $W$ на трактовку пространственных координат в НР пределе требует специального анализа. Но представляет определенный интерес также изучение НP предела при условии $\stackrel{[0]}{W}=W$, в частности в предположении, что релятивистское решение 
задано в (нефизическом) евклидовом 3-пространстве $V_{3}$ в вейлевых координатах $W=\varrho$, которые принимаются попросту переходящими в цилиндрические координаты в предельном (уже физическом) $\stackrel{(0)}{V}$. В данной статье так и предполагается. (В силу уравнений поля из (6) следует $\mu=\mu \eta+1]+O\left(\eta^{2}\right)$, а для ньютонова предела даже $\stackrel{[1]}{\mu=0,}$ так что в НР пределе эта характеристическая величина искривленного физического 3-пространства в метрической форме (4) исччезает.) Отметим еще, что векторы напряженности НР гравитационного поля в $\stackrel{(0)}{V}_{3}$ определяются величинами $\stackrel{[2]}{\lambda}$ и $\stackrel{[1]}{A}\left[{ }^{5-7}\right]$ :

$$
\vec{e}=-\frac{1}{2} v_{N}^{2} \stackrel{(0)}{\nabla} \lambda, \quad \vec{h}=v_{N}{\stackrel{[0]}{W}-1 \vec{n}_{3}} \times \stackrel{(0)}{\nabla} \stackrel{[1]}{A}=\stackrel{(0)}{\nabla} \stackrel{[1]}{\Psi},
$$

где $\vec{n}_{3}$ - единичный касательный вектор координатной линии $x^{3}$, $\stackrel{[0]}{W}=\varrho=R \sin \theta, \stackrel{(0)}{\nabla}$ - оператор градиента в $\stackrel{(0)}{V}_{3}$.

4-мерные характеристики НР поля задаются в локальных декартовых реперах $\left[{ }^{5-7}\right]$. В рассматриваемом случае полей в эти реперы переходят в пределе $\eta \rightarrow 0$ локальные реперы Минковского, в которых заданы базисные 1-формы (5). Например, реперные компоненты тензора кривизны $\stackrel{[0]}{R}(0)(i)(0)(j), \quad$ определяющие геодезическое отклонение (т. е. приливные силы) в НР пределе в случае покоящихся в данный момент в локальном репере пробных частиц, имеют вид

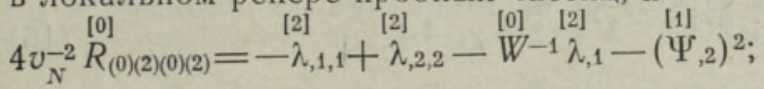

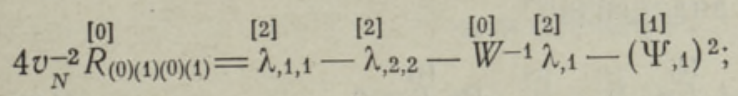

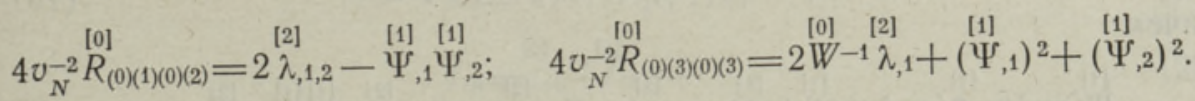

Здесь запятая с индексом означает дифференцирование по цилиндрическим 'координатам $x^{1}=\mathrm{Q}$ и $x^{2}=z$ в предельном $\stackrel{(0)}{V_{3}}$. быть

Для получения ньютонова предела в формулах (8)-(9) должно

$$
\stackrel{(0)}{\nabla} \stackrel{[1]}{A}=\stackrel{(0)}{\nabla} \stackrel{[1]}{\Psi}=0
$$

\section{Общий анализ алгоритмов (1) и (2)}

Прежде всего отметим, что в силу симметричности матриц $\check{\lambda}$ и $\check{\lambda}^{\prime}$ всегда должно иметь место соотношение

$$
\chi_{34}=-\chi_{43} \varepsilon_{+} \varepsilon_{-}+A\left(\chi_{44}-\chi_{33}\right),
$$

а условие $\operatorname{det} \check{\chi}=1$ дает

$$
\chi_{33} \chi_{44}-\chi_{34} \chi_{43}=1
$$

Теперь из алгоритма (2) вытекают формулы 


$$
\lambda^{\prime}=\lambda\left(\chi_{44}+A \chi_{43}\right), \quad A^{\prime}=A-\lambda^{-2} W^{2} \chi_{43}\left(\chi_{44}+A \chi_{33}\right)^{-1} .
$$

Таким образом, при заданном исходном решении можно рассматривать

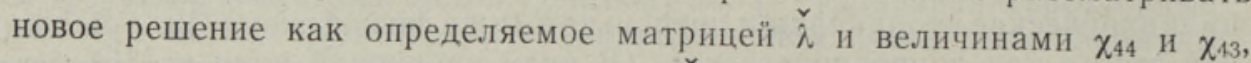
причем остальные элементы матрицы $\chi$ должны удовлетворять соотношениям (11)-(12). Далее с учетом соотношения (1.5), связывающего величину $A$ с твист-потенциалом $\Psi$, можно изучить уже конкретное соответствие между определенными алгоритмами типа (1) и (2). В данной работе ограничимся анализом такого соответствия только в НР пределе.

Будем далее полагать, что как для «нового», так и для «старого» решения имеют место условия (6) или (7). Отсюда следует, что как матрица $\check{\chi}$, так и функционал $F$ должны быть тоже разложимыми по степеням $\eta$. Для низших коэффициентов разложения матрицы $\check{\chi}$ имеем условия

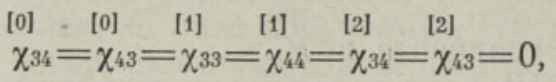

$$
\begin{aligned}
& \stackrel{[0]}{\chi_{44}}=\stackrel{[0]}{\chi_{33}}=1, \quad \stackrel{[1]}{\chi}_{34}=-{\stackrel{[1]}{\chi_{43} W^{2}},}_{[0]}{ }^{[2]}=\stackrel{[2]}{\chi_{33}}=-\stackrel{[1]}{\chi_{44}}-\left[\stackrel{[0]}{\chi_{43} W}\right]^{2} .
\end{aligned}
$$

Отличие физического смысла «нового» решения от смысла «старого»

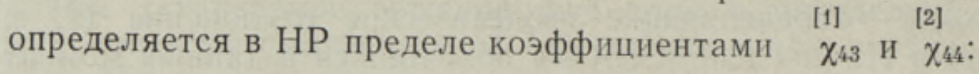

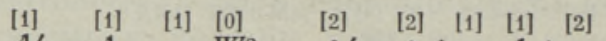

$$
\begin{aligned}
& A^{\prime}=A-\chi_{43} W^{2}, \quad \lambda^{\prime}=\lambda+\chi_{43} A+\chi_{44} \text {. }
\end{aligned}
$$

Для функционала $F$ получим аналогично

причем

$$
F=1+\stackrel{[1]}{F} \eta+\stackrel{[2]}{F} \eta^{2}+\ldots, \quad \operatorname{Re} \stackrel{[1]}{F}=0
$$

$$
\stackrel{[1]}{\Psi^{\prime}} \equiv \operatorname{Im} \stackrel{[1]}{\mathcal{E}^{\prime}}=\operatorname{Im}(\stackrel{[1]}{F}+\stackrel{[1]}{\mathcal{E}}), \quad \stackrel{[2]}{\lambda^{\prime}} \equiv \operatorname{Re} \stackrel{[2]}{\mathcal{E}^{\prime}}=\operatorname{Re}[\stackrel{[2]}{F}+\stackrel{[1]}{F} \mathcal{E}+\stackrel{[2]}{\mathcal{E}}]
$$

Теперь с учетом (13) из сравнения величин (15) и (17) получается взаимоотношение матрицы $\check{\chi}$ и функционала $F$ в общем случае НР предела.

Так как в ньютоновом пределе имеет место (10), то из (15) и (17) получается

$$
\stackrel{[2]}{\lambda^{\prime}} \stackrel{[2]}{=}+\stackrel{[2]}{\lambda}+\stackrel{[2]}{\chi} \underset{44}{\lambda}=\stackrel{\operatorname{Re}}{\stackrel{F}{F}}
$$

отметим, что в силу (8) в данном случае величины $\stackrel{[2]}{\lambda^{\prime}} \stackrel{[2]}{\lambda}$ с точностью до постоянного множителя совпадают с ньютоновыми потенциалами «нового» и «старого» решений соответственно. Из формул (9) видим, что при условии (10) генерация нового решения индуцирует линейный эффект изменения кривизны в НР пределе. Обратим внимание на то, что если получаются новые решения, допускающие неньютонов НР предел, т. е. решения, где вихревая природа поля может сохраняться также при выключении релятивистских эффектов $\left[{ }^{5,8}\right]$, то в силу (17) и (9) генерация таких решений индуцирует нелийнейный эффект изменения кривизны уже в НР пределе. 


\section{Метод Нейгебауэра}

Пусть в алгоритме (1) функционал $F$ имеет вид (П2). Тогда из формулы (16) следуют определенные условия для величин $Q, B$ и $x_{0}$, а затем и для $\varkappa_{k}, Y_{k}$ и $t_{i k}$.

Полагая $Q \neq 0$ (из формул Приложения видно, что противное не представляет интереса), введем новый функционал

$$
M=B Q^{-1} \text {. }
$$

Затем из (П2) и (16) получаются формулы

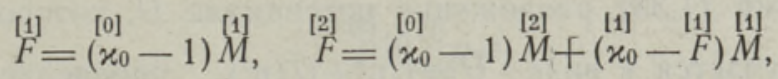

[n]

а для $M$ имеем соотношения

$$
\stackrel{[0]}{M}=0, \quad \stackrel{[1]}{M}=(\stackrel{[0]}{Q})^{-1} \stackrel{[1]}{B}, \quad \stackrel{[2]}{M}=(\stackrel{[0]}{Q})^{-1}(\stackrel{[2]}{B}-\stackrel{\lfloor 1]}{Q} \stackrel{\mid 1]}{M}) .
$$

C точностью до постоянного множителя можно полагать, что $Q$ и $B$ также являются разложениями по степеням $\eta$ типа (16), только из (19) - (20) следует, что должны иметь место условия

$$
\stackrel{[0]}{Q} \neq 0, \quad \stackrel{[0]}{B}=0 .
$$

Конкретные выражения коэффициентов разложений для $Q$ и $B$ получаются с учетом конкретных выражений для этих величин, например, формул (П6) или (П7). В общем случае мы можем только заключить, что и функции $\varkappa_{k}, Y_{k}$ и $t_{i k}$ должны допускать в принципе разложения по степеням $\eta$ :

$$
\begin{aligned}
\varkappa_{k} & ={\stackrel{[0]}{x_{k}}}^{[1]}+\stackrel{x}{*}_{k} \eta+\ldots, \\
Y_{i} & =\stackrel{[0]}{Y}_{i}+\stackrel{[1]}{Y}_{i} \eta+\ldots, \\
t_{i k} & =\stackrel{[0]}{t}_{i k}^{[1]}+{ }^{[1]} t_{i k} \eta+\ldots .
\end{aligned}
$$

Для определения возможных НР аналогов достаточно иметь записанные в формулах $(20)-(21)$ члены разложений для $F$ и $M$. Подчеркнем, что учет членов высших порядков $\stackrel{[3]}{F}, \stackrel{[4]}{F}, \ldots$ и $\stackrel{[3]}{M}, \stackrel{[4]}{M}, \ldots$ позволяет на базе получаемых НР моделей перейти к последовательному выяснению релятивистских поправок в «новых» решениях, генерируемых рассматриваемым методом.

Формулы (19) - (25) и (17) образуют принципиальную вычислительную схему, по которой можно проводить систематический «НР анализ» конкретных вариантов рассматриваемого здесь метода, т. е. анализ конкретных видов функционалов $Q$ и $B$. С одной стороны, мы имеем подход для определения возможных способов введения параметра $\eta$ в конкретные выражения (чтобы получились решения, имеющие НР аналог), а с другой - можем установить, какими именно возможными HP пределами могут обладать новые конкретные решения, получаемые из заданного решения и какие именно «структурные элементы» генерирующего функционала $F$ (постоянные параметры, функциональная зависимость и т. д.) определяют по формулам (8)-(9) динамические характеристики этих полей в предельном 3 -пространстве $\stackrel{(0)}{V_{3}}$ или пре- 
дельную кривизну пространства-времени. При этом четко выделяются сугубо релятивистские аспекты нового решения.

Соображения о «НР анализе», приведенные выше в данном разделе, носили общий характер и фактически не предполагали статичность исходного решения. Далее мы можем принять, что выполняется условие

$$
\varepsilon=\mathrm{e}^{2 U}
$$

и «генерирующие потенциалы» $\boldsymbol{x}_{k}$ определяются формулой (1.29) через функции $\Phi_{k}$ и постоянные $p_{k}, q_{k}$, причем имеет место (23). Поскольку в силу уравнений (1.33) разложение потенциала $U$, которое с учетом (6) и (26) получается в виде $U=\stackrel{[2]}{U} \eta^{2}+O\left(\eta^{3}\right)$, определяет такой же характер разложения функций $\Phi_{k}$, то без органичения общности и с точностью до действительного постоянного множителя можно полагать, что как для числителя, так и для знаменателя в выражении функции $\boldsymbol{x}_{k}(1.29)$ имеем также разложение типа (23), причем

$$
\stackrel{[0]}{x_{k}} \equiv \exp \left(\stackrel{[0]}{i \omega_{k}}\right)=\left(\stackrel{[0]}{q_{k}}-\stackrel{[0]}{i p_{k}}\right)^{2}=\text { const. }
$$

Далее можно убедиться в том, что коэффициент $\stackrel{[1]}{x_{k}}$ является линейной функцией величины $\stackrel{[1]}{\Phi}_{k}$ с определенными постоянными комплексными множителями, а если считать $\stackrel{[1]}{\Phi}_{k}$ исчезающим **, то $\boldsymbol{\varkappa}_{k}$ явля-

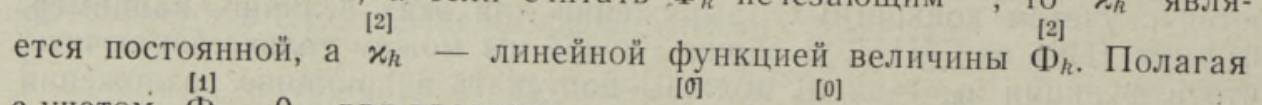
с учетом $\stackrel{[1]}{\Phi_{k}}=0$ для простоты $p_{k}=\stackrel{[0]}{p_{k}}, q_{k}=\stackrel{[0]}{q_{k},}$ имеем

$$
\stackrel{[1]}{x_{k}}=0, \quad \stackrel{[2]}{x_{k}}=i \sin \stackrel{[0]}{\omega_{k}} \exp \left(\stackrel{[0]}{i \omega_{k}}\right) \stackrel{[2]}{\Phi} .
$$

Чтобы установить характер разложений функций $Y_{i}$ и $t_{i k}$, следует с учетом $(21)-(22)$ и $(27)-(28)$ проанализировать конкретные выражения (П6), (П7) и т. д. Такой анализ вместе с выяснением имеющихся возможностей получения НР предела и последующим определением первых релятивистских поправок выходит за рамки данной работы.

В качестве примера рассмотрим здесь только случай ньютонова НР предела и «физического» исходного решения при $N=1$, т. е. с учетом формул (П6) для функционалов $Q$ и $B$. В силу $\stackrel{[1]}{\mathcal{E}^{\prime}}=\stackrel{[1]}{\mathcal{E}}=0$ имеем $\stackrel{[1]}{F}=0$, а отсюда с учетом $(20)$ получаем $\stackrel{[0]}{\left(x_{0}-1\right)} \stackrel{[1]}{M}=0$. Так как в данном случае имеет место (28), то мы должны положить $\boldsymbol{x}_{0} \neq 1$, иначе получилось бы $\stackrel{[2]}{F}=0$ и генерации попросту не было бы. Но с учетом $\stackrel{[0]}{x_{0}} \neq 1$ условие $\stackrel{[1]}{F}=0$ дает $\stackrel{[1]}{M}=0$ и далее в силу

** Как показывает более подробный анализ, для генерирования новых решений, представляющих физический интерес, определенную ценность имеют и такие потенциалы $\Phi_{k}$, в разложениях которых фигурирует неисчезающий член $\stackrel{[1]}{\Phi_{k}}$. Но для получения таких $\Phi_{h}$ мы должны принимать в качестве исходного «нефизическое» решение, при котором $\operatorname{Re} \stackrel{[1]}{\mathcal{E}}=2 U \neq 0$. Такую возможность в данной работе мы затрагивать не
будем. 
имеем $\stackrel{[1]}{B}=0$. Если учесть также условие (22), то из выражения (П6) для $B$ следует, что в разложении (25) первые два члена исчезают, так как из определения (П3) видно, что всегда $\stackrel{[0]}{Y}_{k} \neq 0$. Выбираем еще без ограничения общности $K_{1}$ и $K_{2}$ так, чтобы выполнялось условие $K_{1}+K_{2}=0$. Тогда получаем $t_{2}=\frac{1}{2} t_{21}=-t_{1}, K_{2}=\stackrel{[2]}{K_{2} \eta^{2}}$ и в разложениях для $Y_{1}$ и $Y_{2}: \stackrel{[0]}{Y_{1}}=\stackrel{[0]}{Y_{2}}=1, \stackrel{[11}{Y_{1}}=\stackrel{[11}{Y_{2}}=0 . \quad$ Теперь для рассматриваемого случая имеем

$$
\stackrel{[2 !}{F}=\stackrel{[2]}{=} R^{-1},
$$

где постоянная $\stackrel{[2]}{C}$ имеет вид

$$
\left.\stackrel{[2]}{C}=2 \stackrel{[0]}{2}\left(x_{0}-1\right) \stackrel{[0]}{\left(x_{2}\right.}-\stackrel{[0]}{x_{1}}\right) \stackrel{[2]}{-1} K_{2} .
$$

Так как в силу соотношения (18) величина $\operatorname{Re} \stackrel{[2]}{F}$ пропорциональна «дополнительному» («генерируемому») ньютонову потенциалу, то из полученных соотношений становится совершенно ясно, какой смысл имеет данная операция генерирования нового решения на НР уровне и какой смысл нужно придать постоянным, фигурирующим в этих соотношениях (при этом, очевидно, должно выполняться $\operatorname{Im} \stackrel{[2]}{C}=0$ ).

\section{ПРИЛОЖЕНИЕ}

B $\left[{ }^{2}\right]$ функционал $F$ дан в виде отношения двух определителей

$$
F=D D_{0}^{-1} \text {, }
$$

где $D=\operatorname{det}\left|d_{j k}\right|$, для $j=0: d_{j k}=1$, для $j=2 s: d_{j k}=\gamma_{k}^{8}$, для $j=$ $=2 s-1: d_{j k}=x_{k} \gamma_{k}^{s-1 / 2}, s=1, \ldots, N ; j, k=0,1, \ldots, 2 N$, причем предполагается, что $\gamma_{0}=1$, а $\gamma_{k} \neq 1$, если $k=1,2, \ldots$ Величина $D_{0}$ получается, если в $D$ принимается $x_{0}=1$. В этих формулах $\gamma_{k}$ и $\boldsymbol{x}_{k}$ суть «генерирующие потенциалы», определяемые уравнениями (1.18) и (1.27) соответственно.

С учетом вида функций $\gamma_{k}(1.21)$ и с помощью полезных формул типа формул для величин $\gamma_{l}^{1 / 2}\left(\gamma_{m}-\gamma_{n}\right)\left[{ }^{9}\right]$ функционал $F$ (П1) можно выразить в разных конкретных видах. Нами предлагается подходящий для нашей цели вид

$$
F=\left(Q+\varkappa_{0} B\right)(Q+B)^{-1} .
$$

Здесь $Q$ и $B$ являются определенными комбинациями величин $x_{k}, Y_{k}$ и $t_{i k}(i \neq k), i, k=1,2, \ldots, 2 N$, причем используются следующие определения:

$$
\begin{gathered}
Y_{k}=\left(1-2 t_{k} x+t_{k}^{2}\right)^{-1 / 2}=\sum_{n=0}^{\infty} P_{n}(x) t_{k}^{n}, \\
t_{k}=K_{k} R^{-1}, \quad t_{i k} \equiv t_{i}-t_{k}=\left(K_{i}-K_{k}\right) R^{-1} .
\end{gathered}
$$

$K_{i}$ - постоянные, входящие в выражения (1.21) соответствующих 
функций $\gamma_{k}$, а $R$ и $\theta-$ сферические координаты, введенные в $V_{3}$ вместо вейлевых координат @ и z по формулам

$$
\varrho=R \sin \theta, \quad z=R \cos \theta \quad(x \equiv \cos \theta),
$$

$P_{n}(x)$ - полиномы Лежандра. Подчеркнем, что, как можно показать на основе разложений определителей $D$ и $D_{0}$ в формуле (П1) по первому столбцу, вид функционала $F$ (П2) является совершенно общим. Величины $Q$ и $B$ определяются с точностью до определенного общего множителя, причем их конкретный вид сильно зависит от значения $N$. Если $N=1$, то имеем

$$
Q=\varkappa_{2} Y_{1}-x_{1} Y_{2} \equiv 2 x_{[2} Y_{1]}, \quad B=t_{21} Y_{1} Y_{2} .
$$

Если $N=2$, то получаем

$$
\begin{gathered}
Q=4 x_{[1} Y_{4]} x_{[2} Y_{3]} t_{21} t_{43}+4 x_{[1} Y_{2]} x_{[4} Y_{3]} t_{32} t_{41}, \\
B=\left(x_{2} Y_{1} t_{31} t_{41}-x_{1} Y_{2} t_{32} t_{42}\right) Y_{4} Y_{3} t_{43}+\left(x_{4} Y_{3} t_{31} t_{32}-x_{3} Y_{4} t_{41} t_{42}\right) Y_{2} Y_{1} t_{21} .
\end{gathered}
$$

Отметим, что если положить здесь $x \equiv \exp (i \omega)=$ const, в формуле (П2) $x_{0}=-1$, а в формуле (1) $\varepsilon=1$, то соотношения (П7), (П2) и (1) дают один новый конкретный вид точного решения уравнений Эйнштейна, найденного в $\left[{ }^{9}\right]$. С ростом значения $N$ конкретные выражения $Q$ и $B$ быстро усложняются.

\section{ЛИТЕРАТ У Р А}

1. К о п пе ль А., Изв, АН ЭССР, Физ. Матем. 29, № 4, 364-372 (1980).

2. N e u g e b a ue r, G., J. Phys. A : Math. Gen., 13, № 5, 1737-1740 (1980).

3. Ne u g e b a uer, G., J. Phys. A : Math. Gen., 13, № 2, L19-L21 (1980).

4. Бел инский В. А., 3 а х а ро в В. Е., Ж. эксперим. и теор. физ., 77, вып. 1, 3-19 (1979).

5. Коп пель А., Нерелятивистский анализ релятивистских гравитационных полей, Тарту, Ротапринт ТГУ, 1977.

6. Коппель А., В кн.: Современные проблемы общей теории относительности, Минск, АН БССР, 1979 , с. $48-52$.

7. Koppel, A., In: Abstracts of Contributed Papers for the Discussion Groups. 9th International Conference on General Relativity and Gravitation, July 14-19, 1980, FSU, Jena, GDR, 1980, 1, p. 40-41.

8. К е рес Х., Ж. эксперим. и теор. фнз., 48, вып. 5, 1319-1327 (1965).

9. Kramer, D., Neugebauer, G., Phys. Lett. A., 75, № 4, 259-261 (1980).

Тартуский государственный университет
Поступила в редакцию 14/VII 1980

\section{A. KOPPEL, T. LEMBER}

\section{EINSTEINI VORRANDITE AKSIAALSÖMMEETRILISTE STATSIONAARSETE LAHENDITE GENEREERIMISE MEETODITE MITTERELATIVISTLIKUST ANALUUSIST}

On võrreldud üldistatud kujul Einsteini võrrandite uute täpsete vaakuumlahendite leidmise meetodeid, mida on arendatud artiklites $\left[^{2,3}\right]$ ning $\left[{ }^{4}\right]$, ja esitatud pōhivalemid nende meetodite nn. mitterelativistlikuks analüüsiks. Sellise analüüsi aluseks on võimalike mitterelativistlike analoogide konstrueerimine nii lähtelahendite kui ka genereeritud lahendite jaoks. Funktsioonid, mis iseloomustavad väljavōrrandite lahendeid ja lahendite genereerimise meetodeid, on arendatud astmeridadena dimensioonitu suuruse $\eta=v_{N} c^{-1}$ järgi 
$\left(v_{N}-\right.$ mitterelativistliku piirjuhu teatav karakteristlik parameeter, $c-$ valguse kiirus). On välja selgitatud arenduste need liikmed, mis kirjeldavad uute lahendite genereerimise protseduurist indutseeritud muutusi nii mitterelativistlikule piirjuhule vastava aegruumi kōveruses kui ka mitterelativistliku välja 3-mõõtmelistes karakteristikutes. Artiklites $\left[2,{ }^{3}\right]$ kirjeldatud meetodi tarvis on esitatud arvutusskeem, mille alusel võib teostada nimetatud meetodi erijuhtude süstemaatilist mitterelativistlikku analüüsi ja seejärel, lähtudes leitud välja mudefitest, asuda ka esimeste relativistlike paranduste kindlakstegemisele. Näitena on käsitletud njuutonliku piirjuhu konstrueerimist u' rritava mudeii ühel lihtsamal erijuhul.

\section{A. KOPPEL, T. LEMBER}

\section{ON NON-RELATIVISTIC ANALYSIS OF THE GENERATION METHODS OF AXIALLY SYMMETRIC STATIONARY SOLUTIONS OF EINSTEIN'S EQUATIONS}

Some general forms of the algorithms for generating new exact axisymmetric stationary empty space solutions of Einstein's equations, elaborated in $\left[{ }^{2,3}\right]$ and $\left[{ }^{4}\right]$, are considered, Basic formulae have been presented for comparing these algorithms under themselves and for analyzing their physical meaning (the so-called NR analysis). The analysis is based on the construction of possible non-relativistic (NR) analogs of the initial solutions and of the generated ones. The functions characterizing the solutions of the field equations as well as the generation methods are expanded in power series of a dimensionless quantity $\eta=v_{N} c^{-1}$, where $v_{N}$ is a characteristic parameter of the NR limit, $c$ is the velocity of light. The expansion terms describing the change of the spacetime curvature in the NR limit and the change of the 3-dimensional characteristics of the NR field, induced by the generation of new solutions, have been determined. In the framework of the method developed in $\left[^{2,3}\right]$, a specific calculation scheme is presenter, on the basis of which one can proceed to a systematic NR analysis of particular cases of the method, and further, making use of the obtained NR models, to the discussion of first relativistic corrections. For that purpose some basic relations of the method under consideration have been re-formulated. As an example, the construction of the Newtonian limit has been studied in the case of $N=1$. 